\title{
Síndrome de Burnout entre graduandos de enfermagem em um centro universitário no interior de Pernambuco
}

\author{
Burnout Syndrome among nursing graduates in a university center inside Pernambuco \\ Síndrome de Burnout entre graduandos de enfermería en um centro universitário dentro \\ de Pernambuco
}

Sheila Juliana Leite Lima1, Carlos Thiago Alves ${ }^{1}$, Maria de Fátima Lima Barbosa ${ }^{2}$, Luciana Dilane dos Santos Barbosa ${ }^{3}$, Gabrielly Laís de Andrade Souza ${ }^{4}$, Shirlley Sayonara Bezerra de Melo Torres $^{5}$, Daniela de Aquino Freire ${ }^{2}$, Juliana da Rocha Cabral $^{2}$, Raianne Monteiro Soares ${ }^{6}$, Andreza Cavalcanti Vasconcelos ${ }^{4 *}$.

\section{RESUMO}

Objetivo: Identificar a ocorrência da Síndrome de Burnout nos graduandos de enfermagem em um Centro Universitário no interior de Pernambuco. Métodos: O local da realização da pesquisa foi em um Centro Universitário no interior de Pernambuco, na análise dos dados foi utilizada estatística descritiva, através de distribuições absolutas e percentuais. Para a identificação da síndrome foi utilizado o questionário validado a escala de Burnout de Maslach para estudantes. Para a identificação de burnout foi utilizado o critério de Grunfeld, a síndrome está prevalente quando o sujeito apresentarem alto nível de Exaustão Emocional e/ou alto nível de Despersonalização e/ou baixo nível de Eficácia Profissional. O programa utilizado para digitação dos dados e obtenção dos cálculos estatísticos foi o programa StatisticalPackage for the Social Sciences (SPSS) na versão 15. Resultados: A amostra final foi composta por 184 graduandos de enfermagem. Os resultados mostraram que dos 184 graduandos 177 foram identificados com a síndrome. Conclusão: Concluise que pela demanda que é requerida destes graduandos, apresentou em níveis elevados as dimensões, sinalizando a necessidade de intervenções, favorecendo novas propostas de ensino, melhorando a qualidade e o engajamento do aprendizado.

Palavras-chave: Esgotamento profissional, Burnout, Estresse ocupacional.

\section{ABSTRACT}

Objective: To identify the occurrence of Burnout Syndrome in nursing students at a University Center in the countryside of Pernambuco. Methods: The place where the research was carried out was at the University Center in the interior of Pernambuco, in the data analysis, descriptive statistics were used, through absolute and percentage distributions. Results: The final sample consisted of 184 nursing students. For the identification of the syndrome, the questionnaire validated to the Maslach Burnout scale for students was used. We used the Grunfeld criterion to indicate Syndrome to indicate Burnout when the interviewee has a high level of Emotional Exhaustion or a high level of depersonalization or a low level of personal professional achievement. The results showed that of the 184 undergraduates, 177 were identified with the syndrome. In the data analysis, descriptive statistics was used, through absolute and percentage distributions. The program used to enter data and obtain statistical calculations was the program StatisticalPackage for the Social Sciences (SPSS) in version 15. Conclusion: It is concluded that due to the demand that is required from these graduates, the dimensions presented at high levels, signaling the need for interventions, favoring new teaching proposals, improving the quality and engagement of learning.

Keywords: Professional exhaustion, Burnout, Occupational stress.

${ }^{1}$ Centro Universitário Vale do Ipojuca (UNIFAVIP), Caruaru - PE.

*E-mail: andrezacavalcanti@hotmail.com

2 Universidade de Pernambuco (UPE), Recife - PE.

${ }^{3}$ Instituto Brasileiro de Pós graduação e Extensão (IBPEX), Recife - PE.

${ }^{4}$ Universidade Federal de Pernambuco (UFPE/CAA), Caruaru - PE.

${ }^{5}$ Universidade Federal de Pernambuco (UFPE), Recife - PE.

${ }^{6}$ Faculdade Venda Nova do Imigrante (FAVENI), Venda Nova do Imigrante - ES. 


\section{RESUMEN}

Objetivo: Identificar la aparición del síndrome de Burnout en estudiantes de enfermería en un centro universitario en el interior de Pernambuco. Métodos: El lugar donde se realizó la investigación fue en el Centro Universitario del interior de Pernambuco, en el análisis de los datos se utilizaron estadísticas descriptivas, a través de distribuciones absolutas y porcentuales. Resultados: La muestra final consistió en 184 estudiantes de enfermería. Para la identificación del síndrome, se utilizó el cuestionario validado para la escala de Maslach Burnout para estudiantes. Utilizamos el criterio de Grunfeld para indicar Síndrome para indicar Burnout cuando el entrevistado tiene un alto nivel de Agotamiento Emocional o un alto nivel de despersonalización o un bajo nivel de logro profesional personal. Los resultados mostraron que de 184 estudiantes universitarios, 177 fueron identificados con el síndrome. En el análisis de datos, se utilizaron estadísticas descriptivas, a través de distribuciones absolutas y porcentuales. El programa utilizado para ingresar datos y obtener cálculos estadísticos fue el paquete StatisticalPackage for the Social Sciences (SPSS) en la versión 15. Conclusión: Se concluye que, debido a la demanda exigida por estos graduados, las dimensiones se presentan en niveles altos, lo que indica la necesidad intervenciones, favoreciendo nuevas propuestas docentes, mejorando la calidad y el compromiso del aprendizaje.

Palabras clave: Agotamiento profesional, Burnout, Estrés ocupacional.

\section{INTRODUÇÃO}

Burnout é uma síndrome de esgotamento devido à sobrecarga profissional e emocional, incluindo o relacionamento interpessoal que envolvem as responsabilidades que representam nas características correlativas: exaustão emocional, despersonalização e ineficácia. A exaustão descreve o membro específico, sendo exigido um sentimento ao longe de seus meios. A despersonalização é o elemento interpessoal, em grandes níveis, pode levar a uma impressão principalmente de defesa e proteção, porém com risco de afastamento. A ineficácia é o componente de autoanálise, que geralmente é seguida de sentimentos como a incompetência e o declínio da produtividade (TIRONI MOS, et al., 2016).

A primeira pesquisa científica a respeito de Burnout foi realizada em 1970, pelo psicanalista Herbert Freudenberger, o mesmo desenvolveu uma pesquisa em pessoas jovens que davam apoio à usuários de substância psicoativas, os quais sentiam falta de motivação e prestavam assistência desumana, devido as dificuldades de aceitação ao tratamento dos enfermos. Foi nesse período que a síndrome teve o conceito como exaustão emocional e físico, relacionada ao trabalho. Para obter uma compreensão mais favorável, após algumas mudanças, no decorrer dos anos, acredita-se que está diretamente relacionada às questões interpessoais, nas funções do trabalhador e na qualidade de vida (LOPES FL, et al., 2016).

A tecnologia, atualmente, vem avançando cada vez mais, embora tragam benefícios à sociedade, também interfere de forma significativa no comportamento psicossocial e nas mudanças de comportamento, podendo gerar um impacto considerável na vida do trabalhador (PELEIAS IR, et al., 2017). Para manter o equilíbrio emocional e o bem-estar físico e psíquico, o ser humano desenvolve defesas indesejáveis constantemente, podendo ocasionar distúrbios psicossociais e trabalhos que envolvem condições de sofrimento relacionadas à coletividade e organização. Dessa forma, várias patologias associadas às mudanças introduzidas ao ambiente de trabalho são acometidas pelos profissionais (CAMPOS T, 2015).

As situações estressoras prolongadas resultam na Síndrome de Burnout que passou a ser reconhecida pelo Ministério do trabalho e também pelo Ministério da previdência social, sendo registrada na Classificação Internacional de Doenças, garantindo o afastamento do profissional das suas atividades laborais quando diagnosticada corretamente (SILVA JLL, 2015). Burnout está associada ao trabalho e ao estresse crônico devido ao esgotamento emocional (LOPES FL, et al., 2016).

O estresse ocupacional acomete $70 \%$ dos trabalhadores brasileiros e, desses, $30 \%$ sofrem da Síndrome de Burnout, interferindo de forma significativa na população, gerando um problema de saúde pública devido à alta incidência desta doença ocupacional. Diante das circunstâncias, a síndrome de Burnout é a razão para quais muitos pesquisadores iniciaram estudos com profissionais, especialmente, da área saúde, pelo fato de ter relações intensas de situações estressoras que podem apresentar as dimensões da Síndrome que são a exaustão emocional, a descrença e a eficácia profissional (PROENCI CC, et al., 2017). 
No momento atual, os discentes, assim como os trabalhadores, podem desenvolver a síndrome, bem como a exaustão emocional devido à exigência por parte da docência. Compreendendo essas dimensões, a descrença refere-se ao distanciamento de um comportamento arrogante, e o sentimento de estar sendo incompetente em relação aos estudos que se classifica como a eficácia profissional reduzida (CAMPOS T, 2015).

Estudantes veem, ao ingressar na vida acadêmica, como marco fundamental em seu crescimento profissional, entretanto, encontram vários desafios durante esse período. Conciliar o ambiente de trabalho com atividades nem sempre estabelece o êxito em ambas as partes. O estudante de enfermagem quando já tem uma experiência técnica, as chances de resultados positivos tendem a facilitar seu desempenho. Porém, muitos desses, mesmo com a vivência na área de enfermagem, podem apresentar um rendimento não considerável por motivos de estresse e rotina diária (PELEIAS IR, et al., 2017).

Segundo Sanches IT et al. (2017), durante o período de formação acadêmica, o graduando fica exposto a diversos estressores, onde se observam situações desgastantes, ansiedades e angústias relacionadas aos trabalhos, estágio e futura carreira profissional, posturas inadequadas em sala de aula e no campo de estágio. Estas situações marcam a vida acadêmica, deste futuro profissional, com um emaranhado de sentimentos, como: Insatisfação, dúvida, angústia, ansiedade, medo, raiva, tristeza e insegurança.

Ao se deparar com novas experiências, os estudantes enfrentam obstáculos devido os conflitos interpessoais, a angústia e dúvidas voltadas ao planejamento de sua carreira profissional, o excesso de informações ministradas, os conflitos de lazer e em trabalhos acadêmicos. Além de passar por todos esses desgastes, ainda tem que lidar com os diferentes tipos de patologias (CAMPOS T, 2015).

Face ao exposto, se faz importante investir na prevenção da Síndrome de Burnout em graduandos de enfermagem, pois grande parte desses reside em outros municípios do estado de Pernambuco, além de exercerem atividades laborais conciliando com as atividades acadêmicas e estágios práticos. Considerando as atribuições e carga excessiva de atividades vivenciadas por esses graduandos de enfermagem, surgiu essa pesquisa que objetivou identificar a ocorrência da Síndrome de Burnout nos graduandos de enfermagem.

\section{MÉTODOS}

Foi realizado um estudo descritivo, de coorte transversal e abordagem quantitativa realizado nos meses de março de 2018 a maio de 2018 em um Centro Universitário do Interior de Pernambuco. A amostra foi composta por 184 discentes do Curso Bacharelado em Enfermagem.

Participaram da pesquisa os alunos que estavam regularmente matriculados no primeiro, segundo, nono e décimo período do curso de Enfermagem, nos turnos matutino, vespertino e noturno, que estavam na instituição em seu horário de aula e que assinaram o Termo de Consentimento Livre e Esclarecido (TCLE).

A coleta foi realizada por meio de dois instrumentos, o primeiro com dados sociodemográficos, escolares e laborais, contendo 14 perguntas objetivas de múltiplas escolhas e o segundo com a escala de Burnout de Maslach para estudantes, contendo 15 perguntas de múltipla escolha referentes aos sentimentos / emoções dos estudantes em contexto escolar. As questões são avaliadas em escala likert, variando de 0 (nunca) a 6 (sempre).

Para a identificação de burnout foi utilizado o critério de Grunfeld, a síndrome está prevalente quando o sujeito apresentarem alto nível de Exaustão Emocional e/ou alto nível de Despersonalização e/ou baixo nível de Eficácia Profissional (DIAS KM, et al., 2019).

Os dados foram coletados com total privacidade e anonimato aos participantes. Todo material foi digitado e conferido no programa Microsoft Excel Windows. Analisou-se com base nas medidas de desvio de padrão para construção de tabelas para melhor serem apresentados os resultados da pesquisa.

O desenvolvimento do estudo seguiu as diretrizes da Resolução 466/2012 e 510/16 do Conselho Nacional de Saúde e foi aprovado pelo Comitê de Ética e Pesquisa Centro Universitário do Vale do Ipojuca UNIFAVIP/DEVRY, registrado sob parecer 2.439.754. 


\section{RESULTADOS E DISCUSSÃO}

Dentre os graduandos de enfermagem, 184 concordaram em participar da pesquisa (Tabela 1). Em relação aos dados sociodemográficos, $85,3 \%$ são mulheres, $51,1 \%$ são jovens entre 20 a 25 anos e 75,0\% solteiros. Quando questionados se tinham filhos, 79,9\% afirmaram não ter e a metade da amostra, $50 \%$ relataram residirem em outro município. Segundo o estudo de Proenci CC, et al. (2017), realizado em Curitiba$\mathrm{PR}$, no curso bacharelado em enfermagem, a predominância feminina é muito forte, mesmo após o sexo masculino está incluindo-se a cada dia mais. No estudo de Morégula AA, et al. (2015), realizado também no estado Baiano no município de llhéus, com 72 estudantes de enfermagem, também foi identificado a predominância feminina no curso de Enfermagem.

Corroborando com a temática, Bublitz S, et al. (2015) explicam que a escolha do público jovem pelos cursos de saúde, tendo ênfase a enfermagem, tem se dado, possivelmente, pelo incentivo do governo do país no ingresso ao nível superior de ensino, outro fator que também pode estar envolvido é a fácil absorção do mercado de trabalho para estas áreas e pela remuneração atribuída às classes e por serem muito jovens a grande maioria destes sãos solteiros e não possuem filhos.

Tabela 1 - Distribuição dos graduandos pesquisados, segundo dados sociodemográficos, Caruaru, 2018.

\begin{tabular}{ccc}
\hline Variáveis & № & $\%$ \\
\hline Sexo & 27 & 14,7 \\
Masculino & 157 & 85,3 \\
Feminino & & \\
\hline Idade & 31 & 16,8 \\
Menores de 20 anos & 94 & 51,1 \\
De 20 a 25 anos & 59 & 32,1 \\
Mais de 25 anos & & \\
\hline Estado Civil & 139 & 75,5 \\
Solteiro & 43 & 23,4 \\
Casado ou vivendo junto & 2 & 1,1 \\
Viúvo, separado ou divorciado & & 20,1 \\
Filhos & 37 & \\
\hline Sim & 147 & 59,9 \\
Não & & 50,0 \\
\hline Cidade que reside & 92 &
\end{tabular}

Fonte: LIMA SJL, et al., 2020.

Quando analisado os dados referentes aos estudos, observa-se que em relação ao período que estão matriculados, $20,1 \%$ estão no primeiro período, $7,0 \%$ no segundo, $53,8 \%$ nono e 19,1 estudam atualmente no décimo período. No tocante ao turno que cursam $10,8 \%$ são da manhã, $45,1 \%$ da tarde e $44,1 \%$ do turno da noite. Um dado significante foi em relação aos alunos que trabalham e estudam, pois $60,9 \%$ não trabalham, porém 39,1 afirmaram que além dos estudos exercem uma atividade laboral, destes 5,9\% trabalham em uma jornada de menos de 12 horas semanais, 5,5\% de 12 a 20 horas, $7,1 \%$ de 21 a 30 horas, $10,8 \%$ de 31 a 40 horas e $9,8 \%$ trabalham por mais de 40 horas semanais.

Em relação aos participantes que afirmaram trabalhar, 17,9\% exercem atividade como profissional de enfermagem e a grande parte, $82,1 \%$ não. No tocante aos estudantes que já foram estagiar, a minoria, $24,5 \%$ ainda não realizaram os estágios e 75,5 confirmaram que já fizeram estágios nos campos práticos.

Os graduandos que trabalham e estudam possuem uma vida muito sobrecarregada e desgastante, foi observada a presença desta síndrome em diversos cursos de graduação. O fato desta da jornada trabalho/faculdade acaba afetando o sono e a disposição física e emocional do público em questão, com pouco tempo para conciliar as atividades de rotina, estudar, realizar os trabalhos, assim, torna-se cada vez mais difícil manter o convívio familiar e práticas de lazer, o que aumenta ainda mais a sobrecarga nestes discentes (MOTA ID, et al., 2017). 
Também foi possível avaliar que $48,4 \%$ utilizavam ônibus como meio de transporte de sua residência até a instituição de ensino; $40,8 \%$ dos participantes gastam menos de 30 minutos para chegar à faculdade. Esses fatos evidenciam situações desgastantes, as quais os públicos da pesquisa estão expostos, situações essas que podem corroborar para diversos problemas.

Outro ponto importante é que $57,1 \%$ possuem um tempo disponível para estudar, fora o horário de aula, entre duas e seis horas semanais. Acerca do tempo de sono diário, 46,7\% dormem de 4 a 6 horas por dia. Quando um indivíduo não consegue obter um padrão de sono e repouso adequado ele pode gerar sofrimento mental e estar mais vulnerável ao aparecimento de doenças oportunistas bem como outros problemas de saúde de ordem física ou psicológica, uso de substâncias psicoativas e, até mesmo, o suicídio (GALDINO MJ, et al., 2016).

Dentre os graduandos de enfermagem analisados, 96,2\% foram identificados com a Síndrome de Burnout. Vários pesquisadores evidenciam a Síndrome de Burnout em graduandos, uma vez que estão expostos às três dimensões da síndrome. Vivenciam a exaustão emocional quando surge exaustão decorrente das exigências de estudo, a despersonalização quando há atitudes distanciadas e cínicas em relação ao estudo e a baixa realização quando há a sensação de incompetência como estudante. Agregado a estes também pode ser um fator contribuinte para o desenvolvimento da síndrome a experiência do estágio quando 0 graduando passa a ter que lidar um grande volume de informações, com o planejamento de carreira, com conflitos pessoais e acadêmicos e o lazer, podendo ainda também passar pelo desgaste de lidar com doenças e até com situações de morte (CAMPOS T, 2015).

Esse é um esgotamento devido à sobrecarga profissional e emocional, incluindo o relacionamento interpessoal que envolvem as responsabilidades que representam nas características correlativas: exaustão emocional, despersonalização e ineficácia (TIRONI MOS, et al., 2016).

A Síndrome de Burnout está fragmentada em sintomas que alteram o físico, o psíquico, conduta suscetível de chegar a pessoa de modo individual, organizacional, social, criando questões que afeta a vida do trabalhador, como carga horária excessiva, reconhecimento profissional e remuneração insuficientes principalmente na área da enfermagem fazendo-se indispensável o seu desligamento laboral, à frente desse a empresa percebe a importância em adotar medidas de reduzir a extensão da síndrome acerca dos colaboradores (FERREIRA NN, et al., 2015).

A síndrome é de fácil identificação nos serviços que prestam assistência ao indivíduo como atendimento em saúde, assistência social, saúde mental, profissões relacionadas à religião, aconselhamento, ensino e sistema judiciário, já que estão totalmente ligadas ao fornecimento de ajuda a pessoas necessitadas. Burnout acarreta várias consequências que podem ser observadas, além dos conflitos para o indivíduo, provoca uma deterioração emocional, sociais e reduzindo de forma significativa o desempenho do profissional, causando custos organizacionais elevados (AGUIAR CVN, et al., 2017).

Novos cenários têm sido desenvolvidos em um mundo integrado, com tecnologias, velocidades de produção, tarefas e trabalhadores adaptáveis às novidades, a também uma população esquecida das inovações. O estresse no mundo atual está cada vez mais presente nos indivíduos como no trabalho, se o estresse for crônico e relacionado ao emprego é chamado Síndrome de Burnout, a qual acontece quando o sujeito tem dificuldade de enfrentar cobranças excessivas e conflitos laborais (PINTO TSB, 2017).

Evidencia-se na Figura 1 que se tratando de dimensão de Exaustão, evidencia-se que 72,3\% apresentam nível alto e 17,9\% moderado. Ainda, a exaustão emocional, na qual o esgotamento afeta os trabalhadores, é o primeiro sintoma da síndrome que aparece, como a sensação de esgotamento físico, falta de energia. $\mathrm{Na}$ exaustão emocional os indivíduos sentem o aumento da sobrecarga e meios emocionais enfraquecidos, resultado do contato intenso no cotidiano. Essa dimensão é considerada a característica central e a sua manifestação possibilita a detecção mais facilmente de burnout (JANTSCH N, et al., 2018).

Segundo Giovanne KR (2017), Burnout pode se iniciar ainda na graduação e progredir durante a vida profissional. Sendo assim, é necessário que no diagnóstico seja realizado precocemente, para que a intervenção seja executada o mais cedo possível, evitando repercussões sintomatológicas da Síndrome de Burnout. 
Figura 1 - Frequência percentual dos graduandos de enfermagem quanto à presença da Síndrome de Burnout de acordo com a dimensão Exaustão Emocional (EE), Caruaru, 2018.

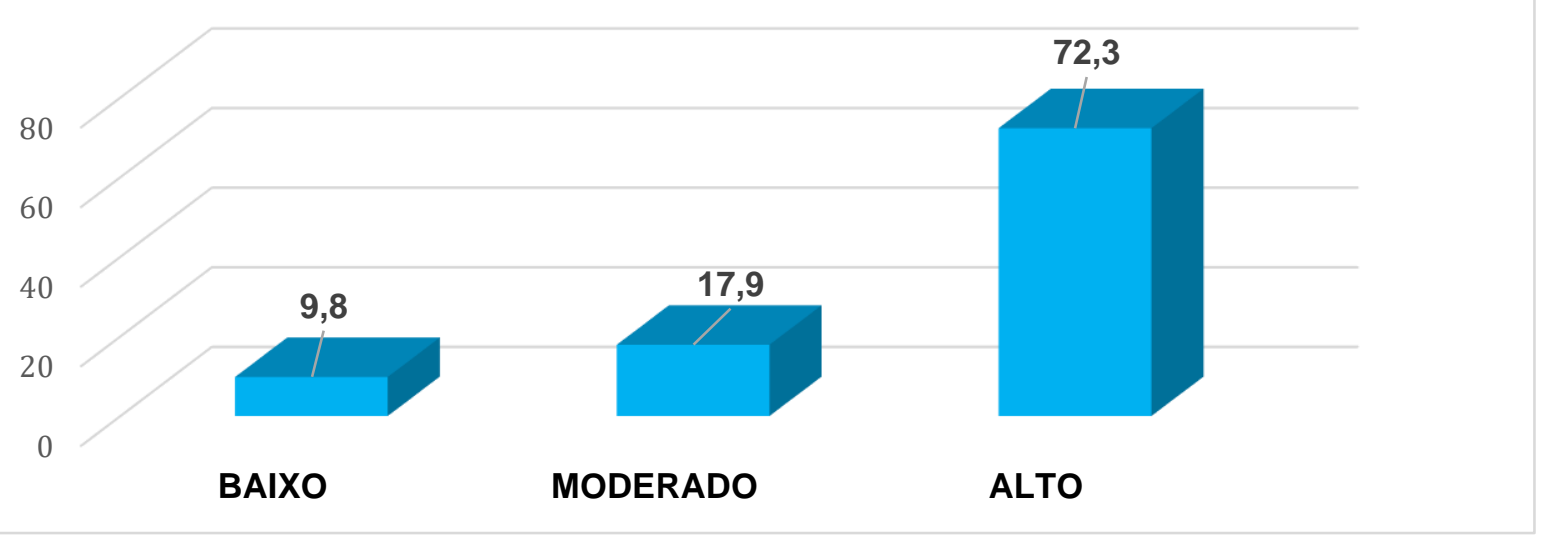

Fonte: LIMA SJL, et al., 2020.

Observa-se, na Figura 2, um predomínio de participantes que apresentam a síndrome em relação à dimensão despersonalização, 39,2\% apresentaram nível moderado. A despersonalização assimila-se com falta de afeto, podendo desfazer vínculo efetivo. O resultado da despersonalização são atitudes e sentimentos negativos, algumas vezes, clínica, de indiferença e indelicadeza com o próximo. Sendo um estado psíquico que prevalece o fingimento efetivo, criticando tudo no meio em que se é integrado socialmente (SOUZA AKS, et al., 2016).

Figura 2 - Frequência percentual dos graduandos de enfermagem quanto à presença da Síndrome de Burnout de acordo com a dimensão Despersonalização (DE), Caruaru, 2018.

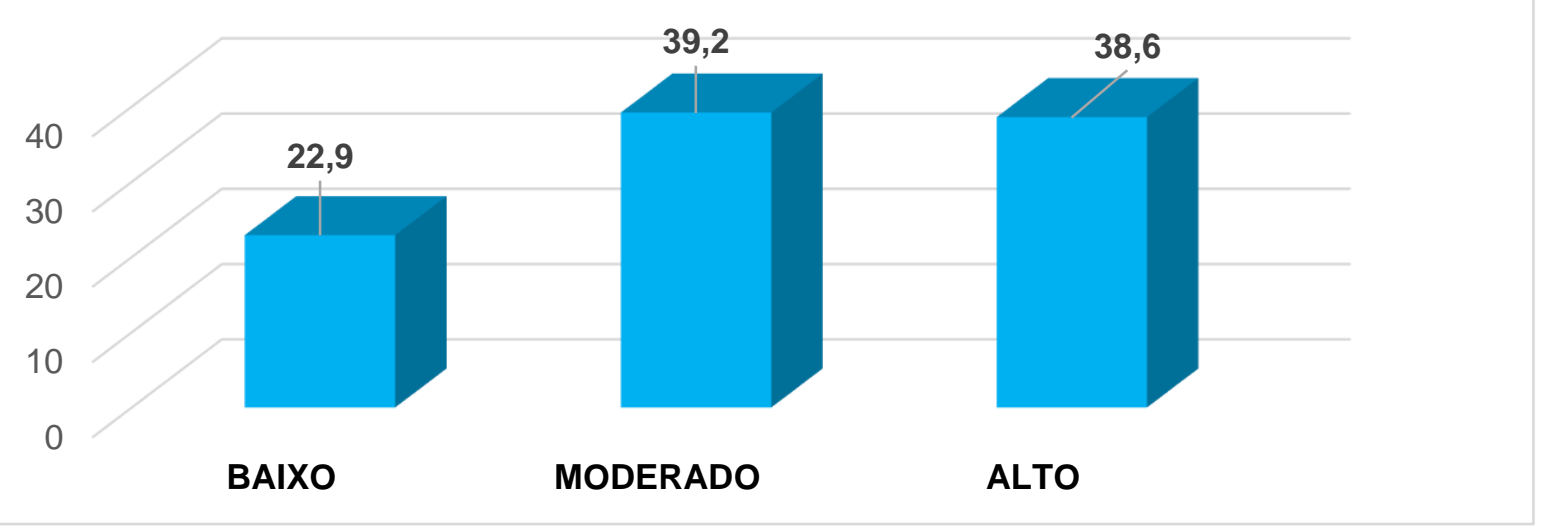

Fonte: LIMA SJL, et al., 2020.

No tocante a dimensão Eficácia Profissional 76,6\% apresentaram nível alto e 14,1\% nível moderado. Nas funções que não tenham um envolvimento com atendimento a clientes, eficácia profissional é uma dimensão que pode ser caracterizada em ter indiferença e atitudes distante da sua ocupação, ao invés das pessoas com as quais se associa com o trabalho (DIAS FS, et al., 2018).

A eficácia profissional são tendências negativas de auto avaliação no trabalho com relação à profissão. Vivências de insuficiência profissional, esgotamento, fracasso, sentimento de vazio, baixa autoestima e impotência também são frequentes. Somados a esta sintomatologia também podemos encontrar inquietude, dificuldade para concentrar-se, irritabilidade, conduta agressiva com companheiros de trabalho e/ou família (SAKAE TM, et al., 2017).

Pesquisa realizada no sul do Brasil por Tomaschewski-Barlem JG, et al. (2014) com 168 estudantes de do curso de graduação de enfermagem mostrou em seus estudos que os alunos estavam satisfeitos e sentiramse eficazes em relação aos estudos. 
Figura 3 - Frequência percentual dos graduandos de enfermagem quanto à presença da Síndrome de Burnout de acordo com a dimensão Eficácia Profissional (EP), Caruaru, 2018.

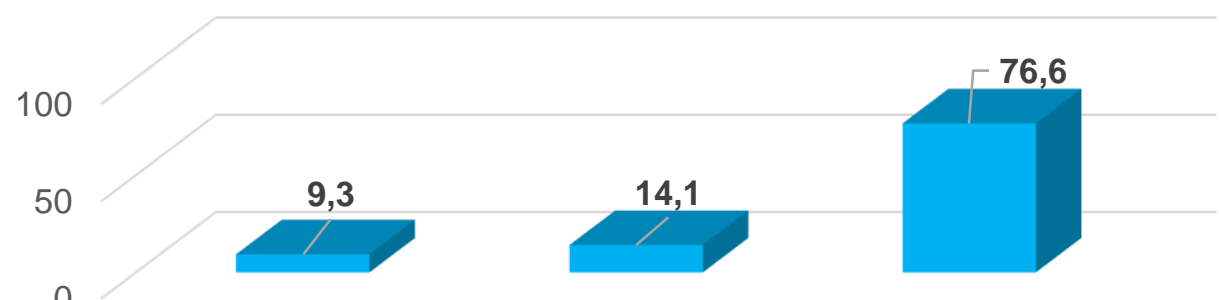

BAIXO MODERADO ALTO

Fonte: LIMA SJL, et al., 2020.

A Tabela 2 descreve os resultados associados aos dados sociodemográficos, laborais e relacionados aos estudos dos participantes que foram identificados com Burnout, é possível constatar que a maiorias com a Síndrome são mulheres, 85,8\%. As mulheres são mais vulneráveis a desenvolver a síndrome, pois, por vezes, conciliam as atividades profissionais com a vida familiar, em distintas atividades tais como: trabalho, contato com enfermidade, relação com familiares, ausência de reconhecimento profissional, sobrecarga no trabalho, falta de autonomia para facilitar a tomada de decisões na função (JANTSCH N, et al., 2018).

Quanto à faixa etária, 51,4\% possuía idade entre 20 e 25 anos. Com relação ao estado civil, observa-se predominância em solteiros $74,6 \%$ e os casados. Sobre o convívio matrimonial, é necessária uma avaliação que seja mais precisa que possa analisar se existem fatores que estimulam a síndrome, pois não há evidências científicas que comprove essa ligação. Na vida conjugal pode desenvolver o Burnout, mas não é relevante essa situação diante dos sintomas, podendo não haver associação nenhuma com os sintomas (GIOVANNE KR, 2017).

Por conseguinte, $79,1 \%$ não tem filhos, e dos que afirmaram possuir filhos, $70,3 \%$ possuíam 1 . Os residentes no município apresentaram percentual de 51,4\%. Segundo Souza LRS, et al. (2018), estudo semelhante em graduandos de terapia ocupacional a relação de ter filhos e do estado civil, pesquisas relata que pode ser visto como refúgio para o Burnout. Visto que os dados mostram a síndrome sendo prevalente em solteiros e sem filhos.

No tocante atividades laborais, um número muito significante $40,1 \%$ dos detectados com Burnout alegaram que trabalham e desses, 52,1\% tem uma jornada acima de 31 horas semanais, porém, $81,9 \%$ não exercem atividades de enfermagem. Burnout é uma consequência gerada através do estresse crônico no ambiente de trabalho. Na definição teórica tem quatro percepções baseadas na etiologia: ambulatorial, sociopsicológica, associativo, sócio habitacional. A mais aplicada na atualidade é a percepção sociopsicologia (SANTOS CLC, et al., 2017)

O acometimento de doenças mentais ocupacionais pode ser oriundo de vários de fatores negativos, como ambientes de trabalho insatisfatório, que falte motivação e bem-estar ao indivíduo e não só sofrimento, estresse e Burnout que não se tratam exatamente de acontecimentos isolados (PAIVA KCM, et al., 2015).

A enfermagem no seu exercício profissional está suscetível à Síndrome. Diante das suas atividades laborais, ficam expostos a diferentes riscos, sejam eles físicos ou mentais, tendo assim como exercício diário manter o equilíbrio psíquico. Esses vivenciam na sua rotina situações de dor, sofrimento e mortes, condições desfavoráveis de trabalho, jornadas de trabalho exaustivas, números de membros na equipe insuficientes, exposição a riscos biológicos, ergométricos, químicos entre outros (PROENCI CC, et al., 2017).

Em relação aos dados acadêmicos ainda relacionados à associação com os dados dos participantes com a síndrome, $52,6 \%$ estão cursando o nono período e $77,4 \%$ já realizaram atividades em campo de estágios. A prevalência da Síndrome de Burnout entre os alunos de enfermagem de diferentes períodos podem estar relacionadas a vários contextos acadêmicos e atribuições executadas em cada condição (PINTO PS, et al., 2018). 
Segundo Sanches GF, et al. (2017), os graduandos de enfermagem passam por momentos de grande turbulência, principalmente no último ano de formação, onde esse terá uma demanda alta de afazeres acadêmicos, desde aulas, estágio supervisionado, Trabalho de Conclusão de Curso (TCC), avaliações entre outras atividades, as quais irão exigir deste discente tempo e dedicação.

Tabela 2 - Distribuição dos graduandos do curso de graduação de enfermagem identificados com a síndrome de Burnout e a relação com dados sociodemográfico, laborais e estudo ( $n=177)$, Caruaru, 2018.

\begin{tabular}{|c|c|c|}
\hline Variáveis & № & $\%$ \\
\hline \multicolumn{3}{|l|}{ Sexo } \\
\hline Masculino & 25 & 14,2 \\
\hline Feminino & 152 & 85,8 \\
\hline \multicolumn{3}{|l|}{ Idade } \\
\hline$<$ de 20 anos & 27 & 15,3 \\
\hline 20 a 25 anos & 91 & 51,4 \\
\hline Mais de 25 anos & 59 & 33,3 \\
\hline \multicolumn{3}{|l|}{ Estado civil } \\
\hline Solteiro & 132 & 74,6 \\
\hline Casado ou vivendo junto & 43 & 24,3 \\
\hline Viúvo, divorciado ou separado & 2 & 1,1 \\
\hline \multicolumn{3}{|l|}{ Filhos } \\
\hline Não & 140 & 79,1 \\
\hline Sim & 37 & 20,9 \\
\hline \multicolumn{3}{|l|}{ Com filhos } \\
\hline 1 filho & 26 & 70,3 \\
\hline Mais de 2 filhos & 11 & 29,7 \\
\hline \multicolumn{3}{|l|}{ Cidade que reside } \\
\hline Caruaru & 91 & 51,4 \\
\hline Outra cidade & 86 & 48,6 \\
\hline \multicolumn{3}{|l|}{ Trabalha } \\
\hline Não & 106 & 59,9 \\
\hline Sim & 71 & 40,1 \\
\hline \multicolumn{3}{|l|}{ Jornada Semanal de trabalho } \\
\hline Menos de 12 horas & 10 & 14,1 \\
\hline De 12 a 20 horas & 11 & 15,5 \\
\hline De 21 a 30 horas & 13 & 18,3 \\
\hline De 31 a 40 horas & 19 & 26,7 \\
\hline Mais de 40 horas & 18 & 25,4 \\
\hline \multicolumn{3}{|l|}{$\begin{array}{c}\text { Atua como profissional de } \\
\text { enfermagem }\end{array}$} \\
\hline Não & 145 & 81,9 \\
\hline Sim & 32 & 18,1 \\
\hline \multicolumn{3}{|l|}{ Período matriculado } \\
\hline Primeiro período & 32 & 17,4 \\
\hline Segundo período & 13 & 7,1 \\
\hline Nono período & 97 & 52,6 \\
\hline Décimo período & 35 & 19,1 \\
\hline \multicolumn{3}{|l|}{ Estágio Prático } \\
\hline Não & 40 & 22,6 \\
\hline Sim & 137 & 77,4 \\
\hline
\end{tabular}

Fonte: LIMA SJL, et al., 2020. 


\section{CONCLUSÃO}

O estudo evidenciou um número expressivo de estudantes com a síndrome de Burnout, desses, a prevalência foram mulheres, na faixa entre 20 e 25 anos, solteiros e sem filho. Uma elevada quantidade de alunos apresentou as dimensões exaustão emocional e despersonalização em níveis elevados, seguida já do nível moderado e eficácia profissional moderado. Com esses resultados, vale salientar a importância de atender à necessidade dessas pessoas que estão com a síndrome ou próximos a desenvolvê-la através de intervenções eficazes. Por conseguinte, espera-se que os achados possam servir de parâmetro para as instituições de ensino superior, a fim de subsidiarem planejamento e estratégias para os graduandos, favorecendo novas propostas pedagógicas de ensino-aprendizagem e com isso favorecendo uma melhor qualidade de vida do público em questão.

\section{REFERÊNCIAS}

1. AGUIAR CVN, et al. Cultura organizacional e adoecimento no trabalho: uma revisão sobre relações entre cultura, Burnout e estresse ocupacional. Psicologia, diversidade e saúde, Salvador - BA, 2017; 6(2):121-131

2. BUBLITZ S, et al. Perfil sociodemográfico e acadêmico de discentes de enfermagem de quatro instituições brasileiras. Revista Gaúcha de Enfermagem, Porto Alegre - RS, 2015; 36 (1):77 - 83.

3. CAMPOS T. Síndrome de Burnout em acadêmicos de enfermagem. Trabalho de Conclusão de Curso (Graduação Em enfermagem). Faculdade de ciências da saúde, Brasília - DF, 2015, 16 p.

4. DIAS FS, ANGÉLICO AP. Síndrome de burnout em trabalhadores do setor bancário: uma revisão de literatura.Trends Psychol. [online], 2018; 26(1): 15-30.

5. FERREIRA NN, LUCCA SR. Síndrome de Burnout em técnicos de enfermagem em um hospital público do estado de São Paulo. Rev Bras Epidemiol, 2015; 18 (1): 68-79.

6. GALDINO MJ, et al. Síndrome de Burnout entre mestrandos e doutorandos em enfermagem. Acta Paul Enferm. 2016; 29 (1):100-106.

7. GIOVANNE KR. Síndrome de burnout em graduandos de enfermagem.(Trabalho de Conclusão de Curso, Bacharelado Em Enfermagem). Faculdade de Ciências da Educação e Saúde-Faces, Brasília - DF, 2017,15 p.

8. JANTSCHN, et al. Síndrome de Burnout: uma revisão integrativa. Research, society and development, 2018; 7(1):0118.

9. LOPES FL, GUIMARÃES GS. Estudo da síndrome de Burnout em estudantes de psicologia. Psicologia: Ensino \& formação, 2016; 7(1): 40-58.

10. MORÉGULA AA, et al. Percepções dos estudantes de enfermagem sobre exposição solar e relação com câncer de pele. Rev Saúde, 2015; 11(4):337-348.

11. MOTA ID, et al. A.Síndrome de Burnout em estudantes universitários: um olhar as investigações. Motrivivência, 2017; 29: 243-256.

12. PAIVA KCM, et al. Estresse ocupacional e síndrome de Burnout: proposição de um modelo integrativo e perspectivas de pesquisa junto a docentes do ensino superior. Rev Unifacs, 2015; 16(3):285-309.

13. PELEIAS IR, et al. A síndrome de Burnout em estudantes de ciências contábeis de IES privadas: pesquisa na cidade de São Paulo. Rev. Repec, 2017; 11 (1):30-51.

14. PINTO TSB. Síndrome de Burnout em docentes. In: Congresso Brasileiro de Ciências da Saúde, Mossoró-RN, 2017, $12 \mathrm{p}$.

15. PINTO PS. et al. Síndrome de burnout em estudantes de odontologia, medicina e enfermagem: uma revisão da literatura. Refacs, 2018; 6 (2):238-248.

16. PROENCI CC. et al. Síndrome de Burnout em trabalhadores da enfermagem que são estudantes da graduação, Revista Saúde e Desenvolvimento, 2017; 11 (6):103-120.

17. SAKAE TM, et al. Prevalência da síndrome de Burnout em funcionários da estratégia da saúde da família em um município no sul do Brasil. Arquivos Catarinenses de Medicina, 2017; 46 (1):p. 43-54.

18. SANCHES GF, et al. Síndrome de Burnout entre concluintes de graduação em enfermagem, Revista de Enfermagem UFPE on line, 2017; 11 (1):31-39.

19. SANTOS CLC, et al. Síndrome de burnout em fisioterapeutas: uma revisão sistemática. Revista pesquisa em fisioterapia, 2016; 7(1):103-114.

20. SILVA JLL. Aspectos psicossociais e síndrome de Burnout entre trabalhadores de enfermagem intensivistas. Tese (Doutorado em Enfermagem) - Escola Nacional de Saúde Pública Sérgio Arouca, Fiocruz, Rio de Janeiro, 2015, 151 p.

21. SOUZA AKS, MARIA AL. Síndrome de burnout em diferentes áreas profissionais e seus efeitos. Rev acta brasileira do movimento humano, 2016; 6(3):1-12.

22. SOUZA LRS, et al. Síndrome de Burnout: mapeamento em graduandos de terapia ocupacional. Rev Ter Ocup Univ, 2018; 29(2):104-110.

23. TIRONI MOS, et al. Prevalência de síndrome de Burnout em médicos intensivistas de cinco capitais brasileiras. BRAS TER INTENSIVA, 2016; 28(3):270-277.

24. TOMASCHEWSKI-BARLEM JG, et al. Síndrome de Burnout entre estudantes de graduação em enfermagem de uma universidade pública. Rev. Latino-Am Enfermagem, 2014; 22(6):934-941. 